\title{
A Precluding Role of Low-Frequency Oscillations for Auditory Perception in a Continuous Processing Mode
}

\author{
Molly J. Henry^ and Björn Herrmann* \\ Max Planck Research Group “Auditory Cognition,” Max Planck Institute for Human Cognitive and Brain Sciences, 04103 Leipzig, Germany \\ Review of Ng et al.
}

Neural oscillatory activity is suggested to play an important role in human perception and cognition (Buzsáki and Draguhn, 2004). For example, recent evidence, largely derived from experiments conducted in the visual domain, suggests that near-threshold target stimuli are better detected when target presentation coincides with the preferred phase in the ongoing neural oscillation. Phase effects on detection performance have been observed in the theta and alpha frequency bands (for review, see VanRullen et al., 2011).

The relation of neural oscillatory phase to auditory perception has been investigated using mostly supra-threshold stimuli (Lakatos et al., 2005; Stefanics et al., 2010). Endogenous oscillations in the delta band were entrained by (i.e., phase locked to) external rhythmic stimulation, leading to precise time locking of the optimal neural delta phase to the onset time of a predictable target. In turn, targets falling into the preferred or "best" phase of the delta oscillation were responded to

Received Sept. 19, 2012; revised 0ct. 4, 2012; accepted 0ct. 8, 2012.

M.J.H. and B.H. are supported by the Max Planck Society (Max Planck Research Group Grant to Jonas Obleser). We are grateful to Benedict Ng for providing us with an example stimulus from the original study.

${ }^{*}$ M.J.H. and B.H. contributed equally to this work.

Correspondence should be addressed to either Björn Herrmann or Molly J. Henry, Max Planck Research Group Auditory Cognition, Max Planck Institute for Human Cognitive and Brain Sciences, Stephanstraße 1A, 04103 Leipzig, Germany. E-mail: bherrmann@cbs.mpg.de or henry@cbs. mpg.de.

DOI:10.1523/JNEUROSCI.4456-12.2012

Copyright $\odot 2012$ the authors $\quad 0270-6474 / 12 / 3217525-03 \$ 15.00 / 0$ more quickly and elicited larger evoked brain responses.

A recent report in The Journal of $\mathrm{Neu}$ roscience was the first to show neural phase effects on the detection of nearthreshold auditory stimuli ( $\mathrm{Ng}$ et al., 2012a). Electroencephalograms (EEGs) were recorded while human participants monitored for a near-threshold target sound embedded in an ongoing stimulus. Specifically, Ng et al. (2012a) created 4-slong stimuli by overlapping and adding 80 different natural sound segments, each 1.4-2 $\mathrm{s}$ in length. A brief target sound (a camera shutter) was presented on each trial at one of six time points distributed across the middle $1 \mathrm{~s}$ section of the stimulus (Ng et al., 2012a; Fig. 1). Participants indicated detection via button press. Behavioral performance showed that participants perceived targets in approximately half of the trials. Trials were thus split into hits and misses for the EEG analysis.

The EEG analyses focused on oscillatory activity in the $2-6 \mathrm{~Hz}$ frequency band. The authors reported results in terms of pre-target power, intertrial phase coherence (ITPC), and a bifurcation index. Power reflects the overall magnitude of oscillatory brain activity, and ITPC indexes the concentration of the neural phase or "phase consistency" across trials. For the bifurcation index, negative values indicate stronger phase concentration in one condition (i.e., hits vs misses), while positive values indicate phase consistency for both hits and misses, but with different preferred phase angles.
Consistent results were observed across measures, with significant differences observed between hits and misses in pre-target time intervals for power, ITPC, and the bifurcation index. Crucial to the authors' main argument, pretarget power and phase consistency across trials (i.e., ITPC) were both larger for misses than for hits. Similarly, the bifurcation index was significantly negative, indicating a more concentrated phase distribution for misses than for hits just before target occurrence. These findings were flanked by an analysis of the instantaneous neural phase at a pre-target time point $(-200 \mathrm{~ms})$, which revealed stronger modulation of performance by pre-stimulus phase for trials in which participants failed to detect the target sound than for trials in which the target was detected.

Thus, this is the first study to demonstrate a specific modulation of miss rates, but not hit rates, for near-threshold stimuli as a function of the phase of ongoing neural oscillations. On this basis, the authors conclude that the role of a lowfrequency $(2-6 \mathrm{~Hz})$ oscillatory phase in audition is a "precluding" rather than an "ensuring" one. Put differently, they suggest that oscillatory phase serves a protective or gating role-keeping out sensory information that coincides with the suboptimal phase of the oscillation rather than selecting sensory information that coincides with the optimal phase of the oscillation. This finding contrasts with a number of previous studies that report 


\section{Example from $\mathrm{Ng}$ et al. 2012}
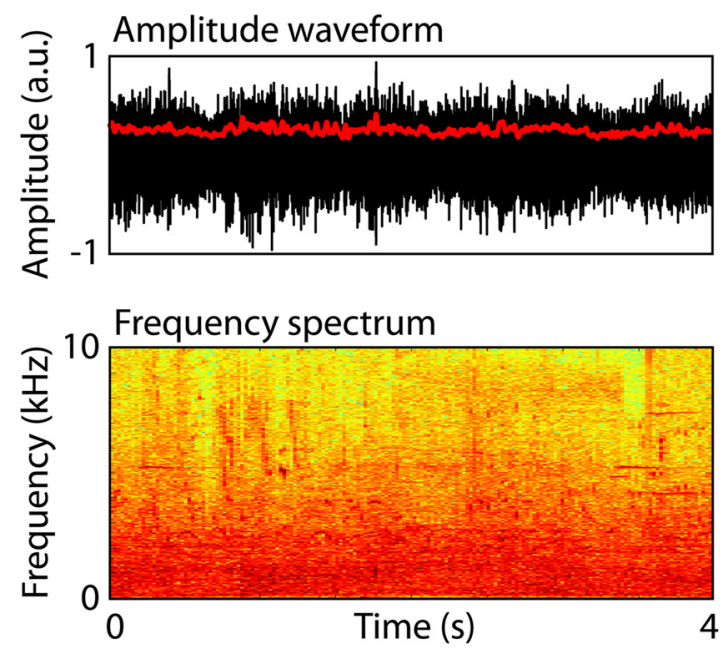
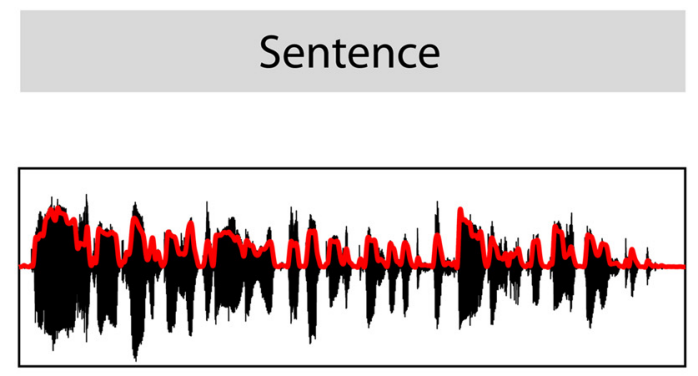

$-80$

Power spectral density (a.u.)

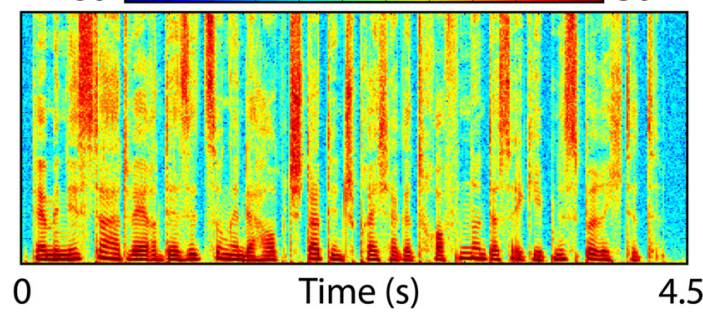

Figure 1._Waveform (top), amplitude envelope (red line), and spectrum (bottom) are shown separately for an example stimulus from the original study (left, courtesy of Benedict Ng), and also a spoken German sentence for comparison (right, "Der Minister hat während der Sitzung in der Hauptstadt den Sprecher getroffen und das Ergebnis berichtet."). In contrast to the temporal regularity contained in the sentence material, the example stimulus possesses no temporal regularity by which neural oscillations could be entrained. (a.u., arbitrary units).

both a "best" and a "worst" phase within the cycle of a low-frequency oscillation (Lakatos et al., 2005; Mathewson et al., 2009).

We suggest that the reason for this discrepancy is the likely use of a continuous (rather than rhythmic) processing mode by listeners in the study of $\mathrm{Ng}$ et al. (2012a). The authors examined brain responses in the $2-6 \mathrm{~Hz}$ frequency range because ITPC in low frequency bands $(<8 \mathrm{~Hz})$ was reported to be greater than in higher frequency bands $(>8 \mathrm{~Hz}$; see $\mathrm{Ng}$ et al., 2012a, their Fig. 2A). In particular, this increased ITPC was interpreted as evidence of neural entrainment. In the context of the brain-environment relation, entrainment involves coupling of ongoing neural oscillations to rhythmic (i.e., oscillatory) external stimulation such that the two systems have, on average, equivalent periods and their phase relationship is stable. By this definition, both oscillatory systems must possess temporal regularity (Roenneberg et al., 2005). Even by a more loose definition of entrainment as involving a neural response that tracks a quasiperiodic signal such as speech over time (Peelle and Davis, 2012), the signal must possess some modulation by which the brain can be entrained.

In contrast, stimuli presented in the study by $\mathrm{Ng}$ et al. (2012a) lacked temporal regularity and thus could not entrain ongoing neural oscillations. Figure 1 depicts a representative example stimulus from the study and shows that, in contrast to the spoken sentence shown for comparison purposes, any low frequency temporal regularity contained in the original sound segments was lost as a result of overlapping and adding 80 sound tokens. In this regard, it is important to note that the reported ITPC data suggesting entrained neural responses (Ng et al., 2012a, their Fig. $2 A$ ) were taken from a previous study by the same group (Ng et al., 2012b) that critically involved a different stimulus in which temporal structure might have supported neural phase locking. In the current study, however, there is insufficient evidence to conclude that the stimulation elicited consistent brain responses that could be taken as entrained.

We therefore suggest that the absence of any "ensuring" effect of a low-frequency oscillatory phase might lie directly in the failure of the stimulation to entrain ongoing brain oscillations. This point is critical in light of the recently proposed distinction between "rhythmic" versus "continuous" processing modes (Schroeder and Lakatos, 2009). Stimuli containing temporal regularities at different time scales are preferably processed in a "rhythmic" mode. This mode supports predictions about the time of occurrence of an upcoming (and possibly important) event. An advantageous and energy-efficient consequence of entrainment to a temporally predictable sequence is that the high-excitability phase of the entrained oscillation aligns with event onsets, making them more likely to elicit a neural response. This corresponds to a "best" neural phase in which detection performance is relatively high. The low-excitability phase, on the other hand, coincides with times at which important events are not expected. This corre- sponds to a "worst," or in the terms of Ng et al. (2012a), "precluding" phase.

As opposed to the rhythmic processing mode, listeners are likely to enter a continuous processing mode when stimuli are temporally unstructured as in $\mathrm{Ng}$ et al., 2012a. During continuous processing, low-frequency modulations are suppressed and long periods of low excitability are minimized (Schroeder and Lakatos, 2009). That is, the low-frequency oscillation spends relatively more time in the high-excitability phase-since the onset time of a future stimulus is unpredictable, it is advantageous to be ready at all times. Thus, a "best" phase for target detection is not necessarily expected. However, a "worst" (i.e., "precluding") phase for detection is likely to be observed during inevitable lapses in the maintenance of high excitability. We suggest that it is exactly these lapses into the low-excitability phase of the low-frequency oscillation that $\mathrm{Ng}$ et al. (2012a) observed and that correspond to the peak in the distribution of misses.

In conclusion, our interpretation of the data of $\mathrm{Ng}$ and colleagues is not at all inconsistent with the existence of both an optimal (excitatory) and a suboptimal (inhibitory) phase of low-frequency oscillations-that is, an "ensuring" and a "precluding" role for low-frequency oscillatory phase. However, we suggest that the temporally unstructured stimulation likely pushed listeners into a continuous processing mode in an effort to reduce the number of targets falling into the "precluding" phase of the oscillation. The result is the observation of a phase angle in which 
misses are maximal; however, there is little modulation of hit rates by phase. In this regard, the results of $\mathrm{Ng}$ and colleagues provide an important piece of support for a continuous processing mode and the first link between neural activity and behavioral consequences during continuous processing in the auditory modality.

\section{References}

Buzsáki G, Draguhn A (2004) Neuronal oscillations in cortical networks. Science 304:19261929. CrossRef Medline

Lakatos P, Shah AS, Knuth KH, Ulbert I, Karmos G, Schroeder CE (2005) An oscillatory hierarchy controlling neuronal excitability and stimulus processing in auditory cortex. J Neurophysiol 94:1904-1911. CrossRef Medline

Mathewson KE, Gratton G, Fabiani M, Beck DM, Ro T (2009) To see or not to see: prestimulus phase predicts visual awareness. J Neurosci 29:2725-2732. CrossRef Medline

Ng BSW, Schroeder T, Kayser C (2012a) A precluding but not ensuring role of entrained low-frequency oscillations for auditory perception. J Neurosci 32:12268-12276. CrossRef Medline

Ng BSW, Logothetis NK, Kayser C (2012b) EEG phase patterns reflect the selectivity of neural firing. Cereb Cortex. Advance online publication. Retrieved Feb. 17, 2012. CrossRef Medline

Peelle JE, Davis MH (2012) Neural oscillations carry speech rhythm through to comprehension. Front Psychol 3:320. CrossRef Medline
Roenneberg T, Dragovic Z, Merrow M (2005) Demasking biological oscillators: properties and principles of entrainment exemplified by the Neurospora circadian clock. Proc Nat Acad Sci U S A 102:7742-7747. CrossRef Medline

Schroeder CE, Lakatos P (2009) Low-frequency neuronal oscillations as instruments of sensory selection. Trends Neurosci 32:9-18. CrossRef Medline

Stefanics G, Hangya B, Hernádi I, Winkler I, Lakatos P, Ulbert I (2010) Phase entrainment of human delta oscillations can mediate the effects of expectation on reaction speed. J Neurosci 30:13578-13585. CrossRef Medline

VanRullen R, Busch NA, Drewes J, Dubois J (2011) Ongoing EEG phase as a trial-by-trial predictor of perceptual and attentional variability. Front Psychol 2:60. CrossRef Medline 